\title{
Differential effects of paclitaxel and docetaxel on gap junctions affects their cytotoxicities in transfected HeLa cells
}

\author{
NAN TANG ${ }^{1,2}$, QIN WANG $^{1}$, DENGPAN WU $^{1}$, SUZHI ZHANG $^{1}$, YUAN ZHANG $^{1}$ and LIANG TAO ${ }^{1}$ \\ ${ }^{1}$ Department of Pharmacology, Zhongshan School of Medicine, Sun Yat-Sen University, Guangzhou, Guangdong 510080; \\ ${ }^{2}$ School of Pharmacy, Guangdong Medical College, Dongguan, Guangdong 523808, P.R. China
}

Received January 21, 2013; Accepted May 23, 2013

DOI: $10.3892 / \mathrm{mmr} .2013 .1546$

\begin{abstract}
Gap junctions (GJs) enhance the cytotoxicity of specific cancer chemotherapeutic drugs and therefore, the inhibition of functional GJs may represent a mechanism by which the toxicity of chemotherapeutics in cancer cells can be reduced. In the present study, the effects and mechanisms of paclitaxel and docetaxel on GJ intercellular communication (GJIC) and the modulation of drug cytotoxicity were investigated in HeLa cells that were stably transfected with the connexin $(\mathrm{Cx}) 32$ expression plasmid. Paclitaxel, but not docetaxel, was observed to inhibit dye-coupling through junctional channels. Gating closure rather than the alteration of Cx32 expression or its membrane localization was responsible for the inhibitory action of paclitaxel on GJ function following short-term exposure. The results revealed that the cytotoxicity of paclitaxel or docetaxel increased in the presence of functional GJs compared with that observed when GJIC was suppressed. In addition, paclitaxel-induced downregulation of GJIC decreased the cytotoxicity of paclitaxel in the presence of functional GJs compared with that of docetaxel, which did not affect $\mathrm{Cx} 32$ channels. These observations demonstrated that the differential effects of paclitaxel and docetaxel on GJIC may affect the cytotoxicity of chemotherapeutic drugs. The present study provides a promising new approach to select antineoplastics and improve drug efficacy in carcinoma cells that form GJs.
\end{abstract}

\section{Introduction}

Paclitaxel and docetaxel belong to the taxane family and are based on the backbone structure of baccatin III. These drugs have been widely used as antineoplastic agents for almost two decades and are effective for the treatment of a wide spectrum

Correspondence to: Professor Liang Tao, Department of Pharmacology, Zhongshan School of Medicine, Sun Yat-Sen University, 74 Zhongshan 2nd Road, Guangzhou, Guangdong 510080, P.R. China

E-mail:taol@mail.sysu.edu.cn

Key words: paclitaxel, docetaxel, gap junction, connexin 32 of cancer forms (1-3). However, the development of tumor resistance to these agents has limited their clinical success (4). Therefore, an approach to enhance the cytotoxicities of paclitaxel and docetaxel while minimizing resistance is required.

Gap junctions (GJs) are plasma membrane channels between adjacent cells that may be open or closed (gating function). GJs are composed of two hemichannels, also known as connexons. Each connexon contains six transmembrane connexin $(\mathrm{Cx})$ monomers and docks to its counterpart in the coupled cell membrane to form a GJ channel. GJs provide a direct method of intercellular communication via the transfer of ions, metabolites and other small molecules. GJ intercellular communication (GJIC) is crucial for a number of physiological processes, including cell proliferation, differentiation, synchronization and maintenance of homeostasis $(5,6)$. In addition, a number of studies have demonstrated that GJIC is important for cancer biology (7-9).

Previous studies have demonstrated that the toxicity of cisplatin and oxaliplatin is increased by the presence of GJIC in transformed cell lines $(10,11)$. The enhanced cytotoxicity may be due to the intercellular transmission of a 'death signal' via GJ channels to neighboring cells. This effect has been reported in radiation treatment, where unirradiated cells adjacent to irradiated cells also underwent cell death $(12,13)$. In addition, the engagement of GJs may represent an approach for enhancing the efficacy of cancer therapeutics, whereas the inhibition of GJs is likely to decrease their efficacy.

The sensitivity of human glioblastoma cells to paclitaxel is increased by $\mathrm{Cx} 43$ expression, in part, by downregulation of BCL-2 (14). Paclitaxel has an inhibitory effect on GJ function in lens epithelial cells, while GJIC is augmented by docetaxel in murine salivary gland carcinoma cells $(15,16)$. However, the link between the cytotoxicity of paclitaxel/docetaxel and GJIC has not been determined. In addition, while the structures of paclitaxel and docetaxel exhibit only minor differences (Fig. 1), their effects on junctional channels are distinguishable. The comprehensive mechanisms by which these two agents affect Cx channels remain largely unknown $(14,16)$.

In the present study, functional GJs were found to enhance paclitaxel- and docetaxel-induced cytotoxicity in HeLa cells transfected with the Cx32 gene (Cx32 HeLa cells). Docetaxel has a modest effect on GJIC, while paclitaxel suppresses GJ function in Cx32 HeLa cells. The inhibition of Cx32 channels by paclitaxel was found to be associated, in part, with the 


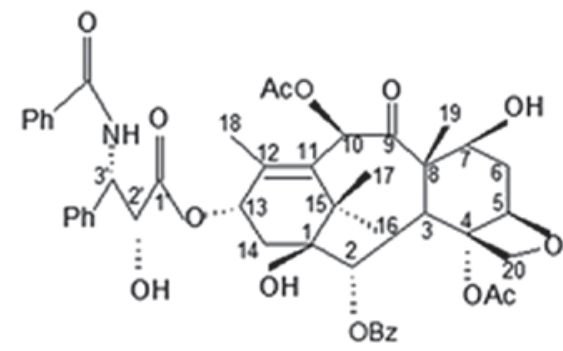

Paclitaxel

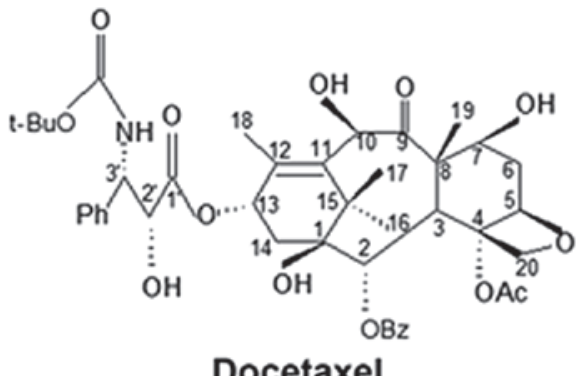

Docetaxel

Figure 1. Structures of paclitaxel and docetaxel.

closure of their gating function following short-term exposure to paclitaxel. The differential effects of paclitaxel and docetaxel on GJs affect their own toxicities in Cx32 HeLa cells.

\section{Materials and methods}

Materials. Paclitaxel, docetaxel, doxycycline and 18 - $\alpha$-glycyrrhetinic acid $(18-\alpha-G A)$ were purchased from Sigma-Aldrich (St. Louis, MO, USA). Calcein acetoxymethyl ester (Calcein-AM) and cell culture reagents were purchased from Invitrogen Life Technologies (Carlsbad, CA, USA). Primary and secondary antibodies for western blot analysis were obtained from Sigma-Aldrich. Secondary antibodies for immunofluorescence were purchased from Invitrogen Life Technologies. All other reagents were purchased from Sigma-Aldrich unless otherwise stated.

Cell culture. The HeLa cell line used in this study expressed the Cx32 gene under the control of a bidirectional tetracycline-inducible promoter and was previously described (17). The Cx32 coding sequence was followed by an influenza hemagglutinin (HA) epitope tag at the C-terminus and Cx32 expression was induced by doxycycline $(1 \mu \mathrm{g} / \mathrm{ml})$ for $48 \mathrm{~h}$. Cells were grown in DMEM supplemented with $10 \%(\mathrm{v} / \mathrm{v})$ fetal bovine serum, $200 \mu \mathrm{g} / \mathrm{ml}$ hygromycin B and $100 \mu \mathrm{g} / \mathrm{ml}$ G418 sulfate.

Paclitaxel/docetaxel and 18- $\alpha-G A$ treatment. Paclitaxel/docetaxel and 18- $\alpha$-GA were dissolved in dimethylsulfoxide (DMSO) and diluted in cell culture medium where required. The final DMSO concentration did not exceed $0.1 \%$ $(\mathrm{v} / \mathrm{v}) .18-\alpha-\mathrm{GA}(10 \mu \mathrm{M})$ was added to the cells $1 \mathrm{~h}$ prior to treatment. 18- $\alpha$-GA was not removed from the cells during paclitaxel/docetaxel treatment.

Assay to measure the cytotoxicity of paclitaxel and docetaxel. The toxicity of paclitaxel/docetaxel was evaluated using a standard colony-forming assay (10). Briefly, cells were seeded at high density $\left(\sim 3 \times 10^{4}\right.$ cells $\left./ \mathrm{cm}^{2}\right)$ to ensure cells were $70-100 \%$ confluent at the time of drug treatment. Following exposure to paclitaxel/docetaxel for $6 \mathrm{~h}$ at $37^{\circ} \mathrm{C}$, cells were rinsed twice with PBS, harvested by trypsinization, counted, diluted and reseeded into 6-well plates at a density of 500 cells/well. The cells were cultured for 5-8 days and then stained with $4 \%$ crystal violet in ethanol. Colonies containing $\geq 50$ cells were counted. For the low cell density condition, cells were seeded at $\sim 100$ cells $/ \mathrm{cm}^{2}$. GJs are not formed under these conditions as the cells were not able to form contacts with each other. Cells were incubated with paclitaxel/docetaxel for $6 \mathrm{~h}$ following attachment and processed as described. Plating efficiency was normalized against the number of colonies formed by vehicle-treated cells. The colony forming efficiency of the untreated cells grown under the low- and high-density conditions were not found to be significantly different from each other (data not shown).

Dye-coupling assay. A 'parachute' dye-coupling assay was used to test the functional GJs, as described previously $(17,18)$. Donor cells were dual-labeled in culture medium supplemented with $5 \mu \mathrm{M}$ CM-Dil and $5 \mu \mathrm{M}$ Calcein-AM. CM-Dil is a red membrane dye that does not permeate through GJs to spread to adjacent cells. Calcein-AM is transformed intracellularly into the GJ-permeable dye calcein. The donor cells were washed with PBS, trypsinized and seeded on a monolayer of receiver cells at a ratio of 1:150 (donor/receiver) for $4 \mathrm{~h}$ at $37^{\circ} \mathrm{C}$. Receiver cells containing calcein were monitored by fluorescence microscopy and the average number of receiver cells containing calcein/donor cell was scored and normalized to that of the control.

Western blot analysis. Western blot analysis was performed as described previously (11). In brief, whole-cell lysates $(20 \mu \mathrm{g})$ were fractionated by $10 \%$ SDS-PAGE and transferred onto a nitrocellulose membrane. Cx32 protein was detected by exposure to mouse anti-HA clone HA-7 IgG in Tris-buffered saline and Tween-20 (dilution, 1:1,000), followed by the addition of alkaline phosphatase-conjugated goat anti-mouse IgG (secondary; dilution, 1:2,000). The anti- $\beta$-actin primary and secondary detection antibodies were diluted to 1:10,000. All western blot exposures were within the linear range of detection and the intensities of immunopositive bands were quantified using Quantity One software (Bio-Rad, Hercules, CA, USA).

Immunofluorescence of $C x 32$. Following treatment with paclitaxel/docetaxel for $1 \mathrm{~h}$, cells were fixed with $4 \%$ paraformaldehyde and then blocked with $2 \%$ BSA. Mouse anti-HA (dilution, 1:200) was applied for $4 \mathrm{~h}$ at room temperature, followed by an FITC-conjugated goat anti-mouse secondary antibody (dilution, 1:400) for $1 \mathrm{~h}$ in the dark. Hoechst 33258 was applied for $5 \mathrm{~min}$ to stain the nuclei of the cells. Following washing three times with PBS, cells were visualized and images were captured using an Olympus IX71 fluorescence microscope.

Dye uptake assay. A dye uptake assay was used for evaluating the hemichannel activity. $\mathrm{Cx} 32 \mathrm{HeLa}$ cells were plated at low densities such that the majority of the cells were not in 
physical contact with each other. The cells were exposed to paclitaxel/docetaxel in $\mathrm{Ca}^{2+}$-free DMEM for $1 \mathrm{~h}$, followed by incubation with $1 \%$ lucifer yellow (LY) and $1 \%$ rhodamine dextran (RD) in PBS for $10 \mathrm{~min}$. LY is a GJ-permeable dye and serves as a tracer for hemichannel activity. RD is too large to spread via GJs and was therefore used as a negative control. Following treatment, the cells were rinsed with culture medium containing normal calcium and observed under a fluorescence microscope. Dye uptake was measured using the percentage of fluorescent cells containing LY normalized to that of the solvent control.

Statistical analysis. Data were analyzed with the Sigma Plot software (Jandel Scientific, San Rafael, CA, USA) using the unpaired Student's t test and presented as the mean \pm SEM. $\mathrm{P}<0.05$ was considered to indicate a statistically significant difference.

\section{Results}

Effect of cell density on the cytotoxicity of paclitaxel/docetaxel. HeLa cells expressing Cx32 were cultured under low-density conditions, in which GJs did not form as the cells were not in physical contact and high-density conditions, which permitted GJ formation. Following exposure to paclitaxel/docetaxel for $6 \mathrm{~h}$, cell survival was assessed using a standard colony formation assay. At the two densities, paclitaxel and docetaxel reduced the clonogenic survival in a dose-dependent manner (Fig. 2). However, the survival of cells plated at a high density was significantly less than that of cells plated at a low density at concentrations of paclitaxel up to $0.1 \mu \mathrm{M}$ (Fig. 2A) or at concentrations of docetaxel up to $0.05 \mu \mathrm{M}$ (Fig. 2B). These results indicate that the cytotoxicity of these agents is greater when cells are plated at high densities. In addition, Fig. $2 \mathrm{C}$ and D demonstrate that the reduction ratios of the cell survival between high- and low-density cultures were $18.5 \pm 3.5,17.7 \pm 2.7,16.8 \pm 4.9$ and $11.11 \pm 2.5 \%$ at concentrations of paclitaxel between 0.01 and $0.5 \mu \mathrm{M}$, respectively and $33.8 \pm 9.0,44.2 \pm 7.7,41.0 \pm 6.4$ and $48.3 \pm 2.0 \%$ at concentrations of docetaxel between 0.001 and $0.05 \mu \mathrm{M}$, respectively. These results indicate that the enhanced sensitivity of docetaxel attributable to the high-density culture is higher than that of paclitaxel at the tested concentrations. Specifically, cell death at low density was $\sim 20 \%$ at $0.05 \mu \mathrm{M}$ paclitaxel and $0.001 \mu \mathrm{M}$ docetaxel. However, cell death at high density was 34 and $49 \%$ at the same concentrations of paclitaxel and docetaxel, which represent increases by factors of 1.7 and 2.5 , respectively. Thus, the toxic effect of docetaxel was higher than that of paclitaxel when cells were grown at high-cell density.

Together, these results demonstrate that the cytotoxicities of paclitaxel and docetaxel are dependent on cell density and indicate that docetaxel induces a greater increase in toxicity to high-density culture than paclitaxel.

Effect of cell density on paclitaxel and docetaxel response is associated with GJIC. The difference between the cytotoxicity of paclitaxel and docetaxel in Cx32 HeLa cells at low- and high-density conditions indicates a possible role for intercellular communication. GJIC is a major pathway for such intercellular communication. To investigate the effect of GJIC on paclitaxel/docetaxel sensitivity, two methods were adopted to modulate $\mathrm{Cx}$ expression and GJ function, including the induction of $\mathrm{Cx} 32$ expression with doxycycline and the inhibition of GJIC using a chemical inhibitor. The induction of Cx32 expression with doxycycline and its localization on the cell membrane were confirmed by western blot analysis and immunofluorescence (Fig. 3A and B). The emergence of GJIC in Cx32 HeLa cells and the inhibitory effect of 18- $\alpha$-GA, a known GJ blocker (19), were detected using a 'parachute' dye-coupling assay (Fig. 3C).

At high densities, the survival of doxycycline-induced (Cx32 expression) cells was substantially decreased at $0.05 \mu \mathrm{M}$ paclitaxel or $0.001 \mu \mathrm{M}$ docetaxel, compared with the survival of uninduced (no Cx32 expression) cells. Specifically, cell survival induced by paclitaxel or docetaxel was significantly reduced by 20.3 and 39.3\%, respectively (Fig. 4A). Incubation of Cx32-expressing cells with $10 \mu \mathrm{M} 18-\alpha-\mathrm{GA}$ under high cell-density conditions increased survival from 65.7 to $79.0 \%$ in the presence of $0.05 \mu \mathrm{M}$ paclitaxel $(\mathrm{P}<0.05)$ and from 51.0 to $81.2 \%$ in the presence of $0.001 \mu \mathrm{M}$ docetaxel $(\mathrm{P}<0.05$; Fig. 4B). These results indicate that the toxicities of paclitaxel and docetaxel are significantly increased when cells are plated at high density and Cx32 is expressed, or when junctional channels are not blocked. These results indicate that enhanced paclitaxel and docetaxel cytotoxicities at high cell densities are mediated by GJIC.

Effect of paclitaxel and docetaxel on GJ function. As described, paclitaxel/docetaxel toxicity is regulated by GJIC at high cell densities. Cell death is likely to markedly reduce the valid cell density for forming GJIC. Therefore, if paclitaxel or docetaxel affected channel function by influencing exclusion from cell death, the toxicity of the agents would be altered. To verify this hypothesis, a 'parachute' dye-coupling assay was performed to determine the effects of paclitaxel and docetaxel on Cx32 channels. Following treatment of Cx32 HeLa cells with paclitaxel/docetaxel for $1 \mathrm{~h}$, which did not lead to cell death, paclitaxel markedly reduced dye coupling (Fig. 4C), while docetaxel had no effect on the spread of dye among cells (Fig. 4D). As demonstrated in Fig. 2, at a concentration range over which the cell density affected paclitaxel/docetaxel toxicity, the sensitivity of docetaxel was higher than that of paclitaxel in the high-density culture (with GJIC) compared with the low-density culture (without GJIC). These observations indicate that the effect of paclitaxel and docetaxel on GJIC may affect their own toxicities.

Effects of paclitaxel and docetaxel on Cx32 expression and its membrane localization. Changes in the number of GJs by affecting $\mathrm{Cx} 32$ expression or its membrane localization is one of the mechanisms by which paclitaxel and docetaxel have been hypothesized to alter GJ function. The expression of Cx32 was determined by western blot analysis. Treatment of $\mathrm{Cx} 32 \mathrm{HeLa}$ cells with a range of paclitaxel/docetaxel concentrations for $1 \mathrm{~h}$ did not alter Cx32 expression levels compared with that of the vehicle control (Fig. 5A).

Following this, $\mathrm{Cx} 32$ localization on the cell membrane was analyzed using immunofluorescence analysis. In control cells (treated with DMSO), Cx32-specific immunoreactivity 
$\mathbf{A}$
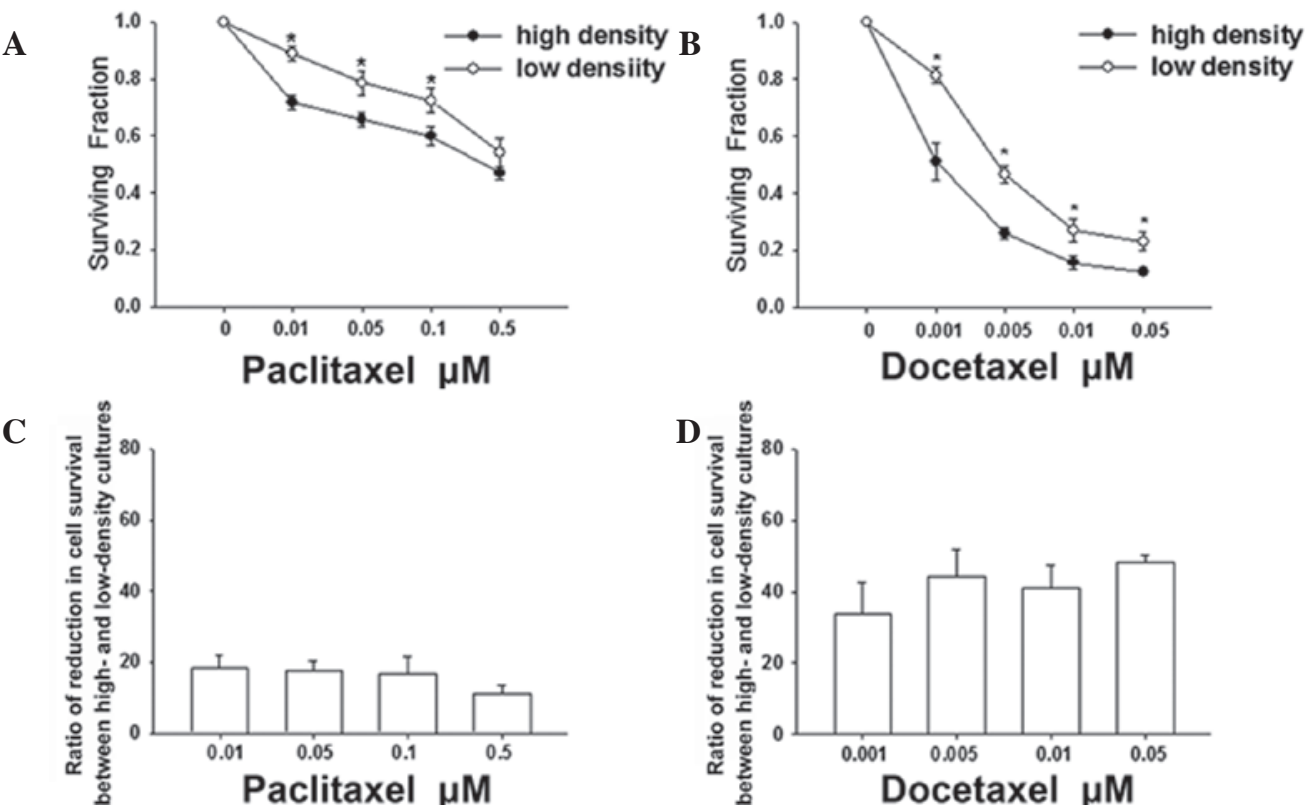

Figure 2. Cytotoxicity of paclitaxel and docetaxel on HeLa cells expressing Cx32 is density dependent. Cell survival was measured by standard colony-forming assay following exposure to a range of (A) paclitaxel or (B) docetaxel concentrations for $6 \mathrm{~h}$ at high- and low cell density. Data are presented as the mean \pm SEM from 4-6 experiments. ${ }^{*} \mathrm{P}<0.05$, vs. high cell density. (C and D) Ratio of reduction in cell survival between high- and low-density cultures. Bars in (C) are presented as the mean \pm SEM from (A). Bars in (D) are presented as the mean \pm SEM from (B). Cx, connexin.

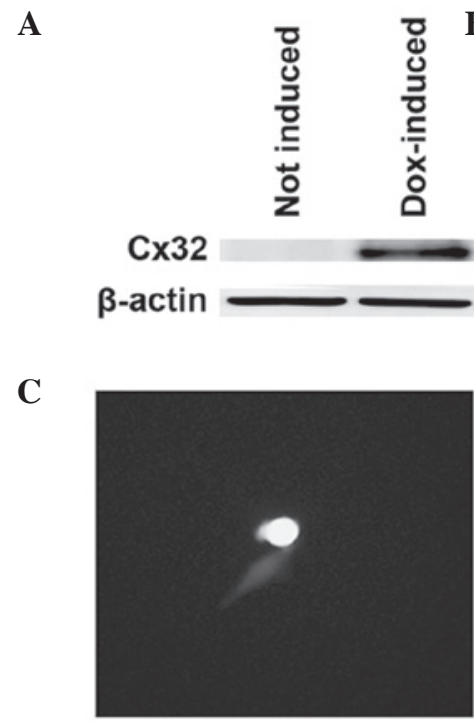

Dox-induced+18- $\alpha-G A$

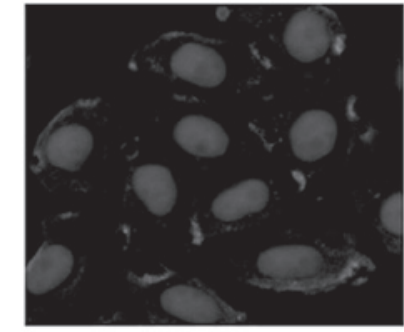

Dox-induced

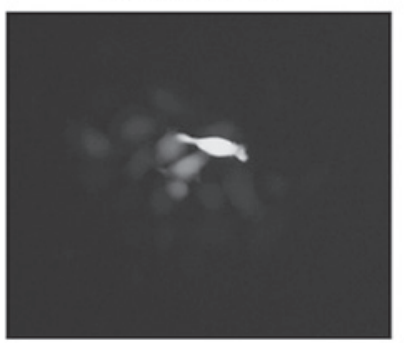

Dox-induced

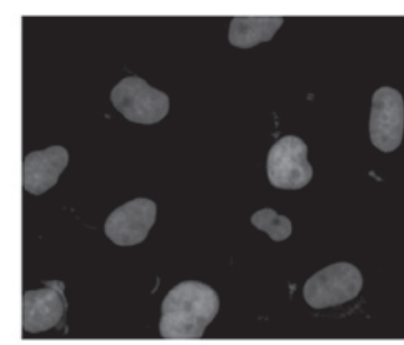

Not induced

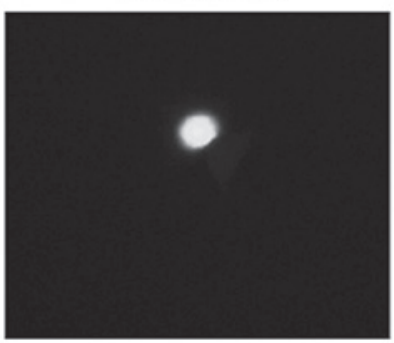

Not induced

Figure 3. Dox induced Cx32 expression, membrane localization and inhibition of the junctional channel by 18- $\alpha$-GA. (A) Western blot analysis of Cx32 expression induced by Dox for $48 \mathrm{~h}$. (B) Localization of Cx32 on the cell membrane of Cx32-transfected and Dox-induced HeLa cells compared with the Cx32-transfected HeLa cells without induction. (C) 18- $\alpha$-GA inhibited dye spread in Cx32 HeLa cells, as demonstrated by the parachute dye-coupling assay. Dox, doxycycline; Cx, connexin; $18-\alpha-\mathrm{GA}, 18-\alpha$-glycyrrhetinic acid.

was predominantly localized to the plasma membrane at cell-cell junctions (Fig. 5B). Cells treated with various concentrations of paclitaxel/docetaxel for $1 \mathrm{~h}$ revealed an almost invariant level of $\mathrm{Cx} 32$ immunoreactive foci at the cell membrane, even at $10 \mu \mathrm{M}$ paclitaxel and $1 \mu \mathrm{M}$ docetaxel (Fig. 5B).

These observations indicate that, following short-term $(1 \mathrm{~h})$ treatment, the effects of paclitaxel/docetaxel on junctional function are not a result of affecting Cx32 expression or its membrane localization.
Effects of paclitaxel and docetaxel on Cx32 hemichannel activity. Treatment with paclitaxel/docetaxel for $1 \mathrm{~h}$ did not alter the expression and localization of $\mathrm{Cx} 32$, indicating that these agents may inhibit dye coupling by affecting channel gating. To test this hypothesis, the permeability of the hemichannel was examined by a dye uptake assay. LY uptake was observed in control Cx32 HeLa cells (Fig. 6A). Dye uptake activity was altered in cells treated with paclitaxel for $1 \mathrm{~h}$. As demonstrated in Fig. 6B, docetaxel did not affect Cx32 hemichannel activity; however, paclitaxel had an inhibitory effect. 
A

C
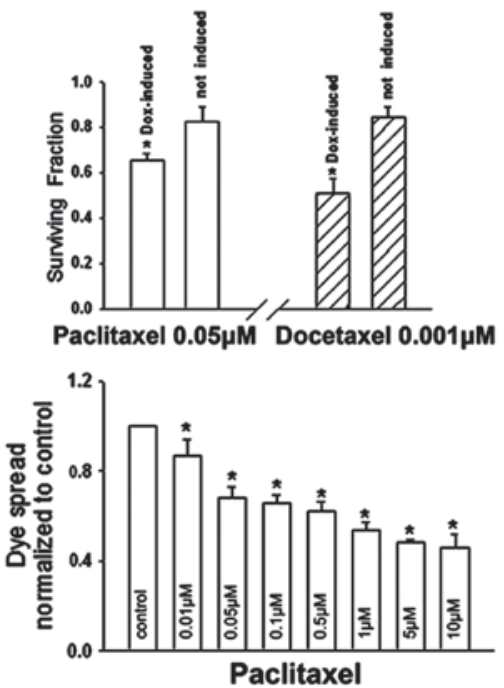

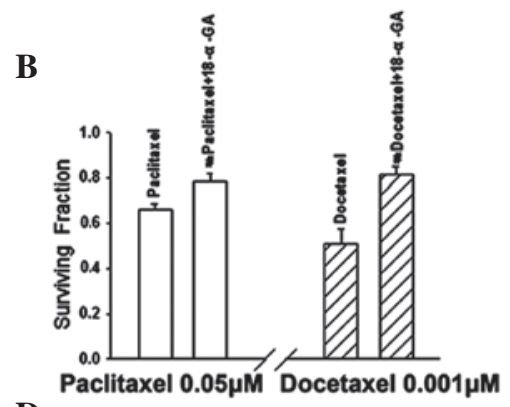

D

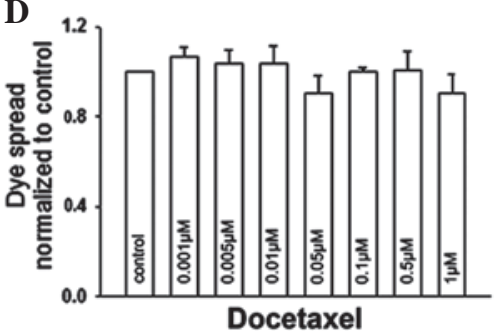

Figure 4. Inhibition of GJIC reduces paclitaxel- and docetaxel-induced cell death and effect on dye coupling in Cx32 HeLa cells. (A) Clonogenic survival of non-Cx32 and Cx32-expressing HeLa cells treated with paclitaxel or docetaxel for $6 \mathrm{~h}$ at high cell density and (B) Cx32 HeLa cells treated with paclitaxel or docetaxel with or without $18-\alpha-\mathrm{GA}$ at high cell density. ${ }^{*} \mathrm{P}<0.05$, vs. non-Cx32; ${ }^{*} \mathrm{P}<0.05$, vs. paclitaxel and docetaxel in (B). (C and D) Cells were treated with various concentrations of paclitaxel and docetaxel for $1 \mathrm{~h}$ and GJIC was evaluated by counting the average number of receiver cells containing calcein per donor cell, normalized against vehicle control. " $\mathrm{P}<0.05$, vs. vehicle control. Data are presented as the mean \pm SEM from four experiments. GJIC, gap junction intracellular communication; Cx, connexin; 18- $\alpha$-GA, 18- $\alpha$-glycyrrhetinic acid.

A
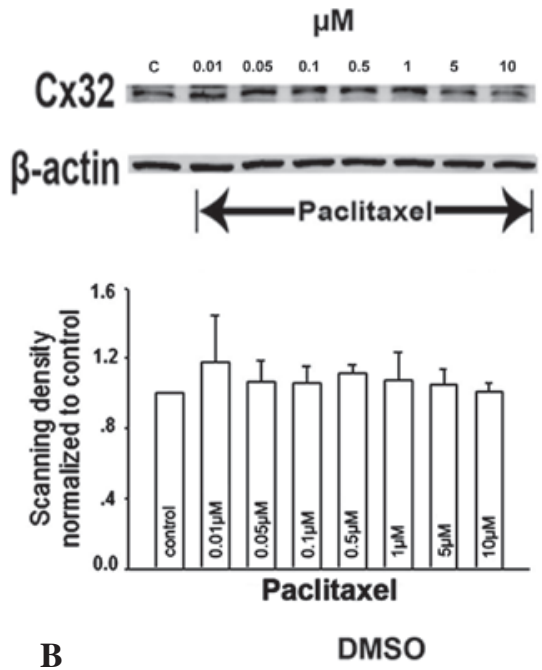

Cx32

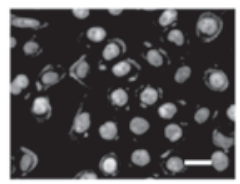

Paclitaxel 10 $\mu \mathrm{M}$
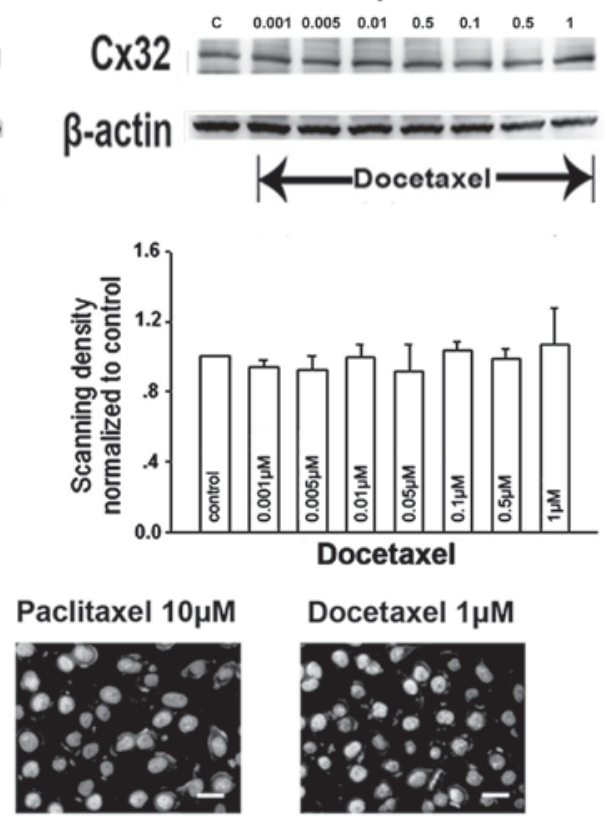

Docetaxel

\section{Docetaxel $1 \mu \mathrm{M}$}

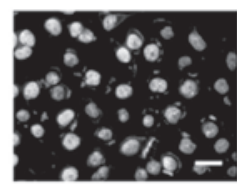

Hoechst 33258
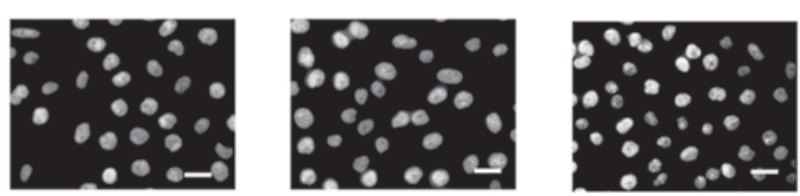

Figure 5. Effects of paclitaxel and docetaxel on Cx32 expression and its membrane localization in Cx32 HeLa cells. (A) Western blot analysis of Cx32 expression following treatment with paclitaxel and docetaxel for $1 \mathrm{~h}$. Data obtained from densitometry of the blots are presented as the mean \pm SEM from three experiments. (B) Localization of Cx32 in cells treated with DMSO (vehicle control), paclitaxel or docetaxel for $1 \mathrm{~h}$ by immunofluorescence. Scale bars, $20 \mu \mathrm{m}$. Cx, connexin.

The results are consistent with the effect of these agents on junctional function (Fig. 4C and D); however, specific values exhibited small deviations. These deviations may be due to differences in the two experimental approaches. Together, the observations demonstrate that paclitaxel-induced closure of 'gating' is largely responsible for its GJIC inhibition. 
A

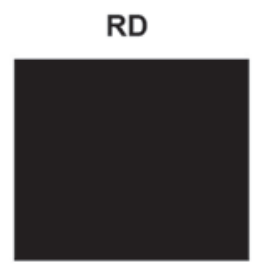

LY

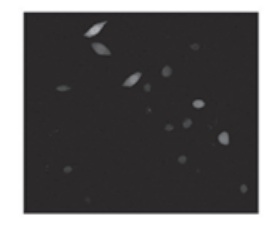

B

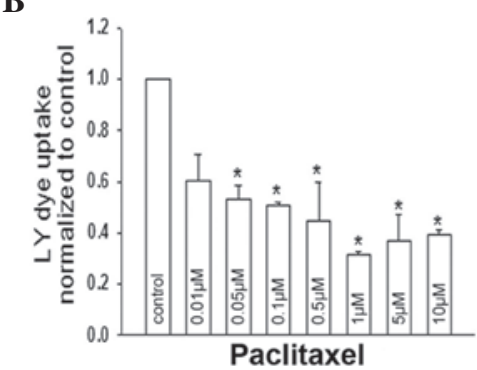

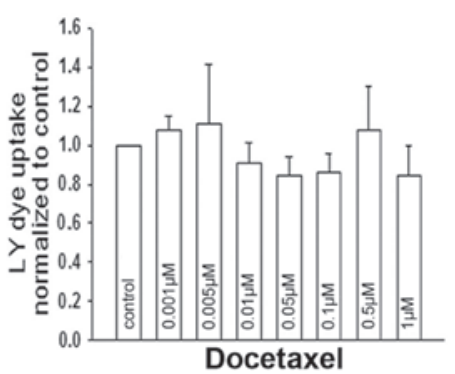

Figure 6. Effect of paclitaxel and docetaxel on hemichannel dye uptake in Cx32 HeLa cells. (A) Cells were cultured at low density and LY uptake was observed following treatment with DMSO (vehicle control) for $1 \mathrm{~h}$. RD was used as a negative control. (B) LY uptake by cells treated with a range of paclitaxel and docetaxel concentrations for $1 \mathrm{~h}$. Dye uptake was assessed by the percentage of LY fluorescent cells, normalized against vehicle control. Data are presented as the mean \pm SEM from three experiments. ${ }^{*} \mathrm{P}<0.05$, vs. vehicle control. Cx, connexin; LY, lucifer yellow; RD, rhodamine dextran.

\section{Discussion}

The present study indicates that GJIC is an important constituent of paclitaxel- and docetaxel-induced cytotoxicity in Cx32 HeLa cells. The toxicity of paclitaxel/docetaxel was increased in cell cultures at high density (i.e., cells contact each other) compared with those at low density (i.e., cells lack junctional contacts). To exclude other potential differences, besides GJIC, which exist when cells are grown at low- vs. high-cell density that may account for the increased cytotoxicity, two methods to suppress the GJs in high-density culture were performed. The results confirm that the activities of paclitaxel and docetaxel were lower when $\mathrm{Cx} 32$ was not induced or when the GJ was blocked by $18-\alpha-\mathrm{GA}$, indicating that $\mathrm{Cx} 32$-composed GJIC may be targeted to enhance the cytotoxicity of paclitaxel and docetaxel.

In addition, these results indicate that paclitaxel inhibits GJIC, which leads to an attenuation of its own cytotoxicity. The two agents have similar toxicities in the absence of GJIC. However, docetaxel is more active than paclitaxel in the presence of GJIC as it does not significantly affect the Cx32 channels, unlike paclitaxel (Fig. 4C and D). Although a number of studies have reported that GJIC is lost in numerous types of carcinoma $(8,20-22)$, the expression of $\mathrm{Cx}$ and GJC are preserved in specific forms of cancer or increase during the invasion and metastatic stages with nominally defective GJs (23-27). In these GJs, which are derived from Cx32 or $\mathrm{Cx} 43$, one must consider the differential effects of paclitaxel/docetaxel on GJIC and how these effects are likely to impact their therapeutic efficacy.

Paclitaxel and docetaxel promote microtubule assembly, inhibit depolymerization and block mitosis in proliferating cells (28). However, their effects on Cx channels are distinguishable. Results of the current study indicate that paclitaxel impaired GJIC; however, this effect was not observed following short-term $(1 \mathrm{~h})$ treatment of Cx32 HeLa cells by docetaxel. In longer-term treatments ( $48 \mathrm{~h}$ ) of various cell lines with $\mathrm{Cx} 43$ derived GJs, paclitaxel suppressed dye spread (15), while docetaxel had the opposite effect (16). Although channels are composed of different $\mathrm{Cx}$ isoforms with diverse permeabilities and sensitivities to regulatory agents (29), paclitaxel and docetaxel treatment had different effects on $\mathrm{Cx} 32$ or $\mathrm{Cx} 43$ channels.

Paclitaxel treatment for $1 \mathrm{~h}$ appears to block Cx32 channels primarily by blocking the gating function, thus, resulting in a closed channel and not by decreasing Cx32 expression or promoting its translocation from the cell membrane to the cytoplasm. Paclitaxel may have a direct effect on channel gating. Cx's are hypothesized to belong to the same family of proteins based on sequence similarity. These proteins have four predominantly transmembrane hydrophobic regions and two extracellular loops with the amino- and carboxyl-terminals (CT) located in the cytoplasm $(5,30)$. Modulators may induce a conformational change in $\mathrm{Cx}$ to directly alter GJIC. For example, quinine blocks $\mathrm{Cx} 36$ and $\mathrm{Cx} 50$ channels by binding to an intracellular site, possibly within the channel pore (31) and 2-APB directly inhibits Cx26 and/or Cx32 channels with the involvement of the CT domain of the Cx (32).

The structural differences of paclitaxel and docetaxel lie in the C3'-substituents of the C13 side chain and an acetyl or a hydroxyl substituted at the $\mathrm{C} 10$ position (Fig. 1). These changes may affect the conformation of $\mathrm{Cx} 32$ to cause gate closure. The differential effects of paclitaxel and docetaxel indicate that the hydrophobic group at the $\beta$-position in the amide group of the $\mathrm{C} 3$ '-substituents in the $\mathrm{C} 13$ side chain that bonds with the conjugated double-bonds (e.g., phenyl) may induce channel inhibition by: i) $\pi-\pi$ stacking interactions with the phenyl rings of aromatic amino acid side chains or the imidazolyl group of histidine or ii) a hydrophobic interaction entering the hydrophobic region of the protein structure. The acetyl (paclitaxel) or hydroxyl (docetaxel) groups at C10 may enhance the interaction with amino acid residues by hydrogen bonding; however, docetaxel had no impact on channel activity. This result indicates that the moiety may not enter the inner part of the protein and is therefore not directly involved in non-covalent interactions with amino acid residues. These results, as well as the structural diversity of taxanes and the conformation of $\mathrm{Cx}$ proteins $(33,34)$ indicate that the specific interactions between taxanes and Cx must be studied further.

Results of the present study indicate that the different actions of paclitaxel and docetaxel on the intercellular communication mediated by GJs that are, in turn, derived from $\mathrm{Cx} 32$, affects their own cytotoxic activities. In addition, short-term exposure to paclitaxel appears to primarily affect the function of the junction by affecting the gating mechanism of the channel. These observations indicate a promising new approach in which the appropriate taxane may be selected for the treatment of cancer based on the presence or absence of GJs in a specific carcinoma. 


\section{Acknowledgements}

The present study was supported by grants from the National Natural Science Foundation of China (nos. 30901807 and 30973434), the Fundamental Research Funds for the Central Universities (no. 10YKPY32) and Grant for Development of Important New Drugs from Xinjiang province, China (no. 201230045).

\section{References}

1. Gelmon K: The taxoids: paclitaxel and docetaxel. Lancet 344: 1267-1272, 1994.

2. King KM, Lupichuk S, Baig L, Webster M, Basi S, Whyte D and Rix S: Optimal use of taxanes in metastatic breast cancer. Curr Oncol 16: 8-20, 2009.

3. Shepherd FA, Dancey J, Ramlau R, et al: Prospective randomized trial of docetaxel versus best supportive care in patients with non-small-cell lung cancer previously treated with platinum-based chemotherapy. J Clin Oncol 18: 2095-2103, 2000

4. Galletti E, Magnani M, Renzulli ML and Botta M: Paclitaxel and docetaxel resistance: molecular mechanisms and development of new generation taxanes. ChemMedChem 2: 920-942, 2007.

5. Maeda S and Tsukihara T: Structure of the gap junction channel and its implications for its biological functions. Cell Mol Life Sci 68: 1115-1129, 2011

6. Vinken M, Vanhaecke T, Papeleu P, Snykers S, Henkens T and Rogiers V: Connexins and their channels in cell growth and cell death. Cell Signal 18: 592-600, 2006.

7. Holder JW, Elmore E and Barrett JC: Gap junction function and cancer. Cancer Res 53: 3475-3485, 1993.

8. Mesnil M, Crespin S, Avanzo JL and Zaidan-Dagli ML: Defective gap junctional intercellular communication in the carcinogenic process. Biochim Biophys Acta 1719: 125-145, 2005.

9. Kandouz M and Batist G: Gap junctions and connexins as therapeutic targets in cancer. Expert Opin Ther Targets 14: 681-692, 2010.

10. Jensen R and Glazer PM: Cell-interdependent cisplatin killing by $\mathrm{Ku} / \mathrm{DNA}$-dependent protein kinase signaling transduced through gap junctions. Proc Natl Acad Sci USA 101: 6134-6139, 2004.

11. Wang Q, You T, Yuan D, et al: Cisplatin and oxaliplatin inhibit gap junctional communication by direct action and by reduction of connexin expression, thereby counteracting cytotoxic efficacy. J Pharmacol Exp Ther 333: 903-911, 2010.

12. Prise KM and O'Sullivan JM: Radiation-induced bystander signalling in cancer therapy. Nat Rev Cancer 9: 351-360, 2009.

13. Harada K, Nonaka T, Hamada N, et al: Heavy-ion-induced bystander killing of human lung cancer cells: role of gap junctional intercellular communication. Cancer Sci 100: 684-688, 2009.

14. Huang RP, Hossain MZ, Huang R, Gano J, Fan Y and Boynton AL: Connexin 43 (cx43) enhances chemotherapy-induced apoptosis in human glioblastoma cells. Int J Cancer 92: 130-138, 2001.

15. Giessmann D, Theiss C, Breipohl W and Meller K: Decreased gap junctional communication in neurobiotin microinjected lens epithelial cells after taxol treatment. Anat Embryol (Berl) 209: 391-400, 2005.

16. Piechocki MP, Lonardo F, Ensley JF, Nguyen T, Kim H and Yoo GH: Anticancer activity of docetaxel in murine salivary gland carcinoma. Clin Cancer Res 8: 870-877, 2002.
17. Koreen IV, Elsayed WA, Liu YJ and Harris AL: Tetracycline-regulated expression enables purification and functional analysis of recombinant connexin channels from mammalian cells. Biochem J 383: 111-119, 2004.

18. Goldberg GS, Bechberger JF and Naus CC: A preloading method of evaluating gap junctional communication by fluorescent dye transfer. Biotechniques 18: 490-497, 1995.

19. Davidson JS, Baumgarten IM and Harley EH: Reversible inhibition of intercellular junctional communication by glycyrrhetinic acid. Biochem Biophys Res Commun 134: 29-36, 1986.

20. Loewenstein WR and Kanno Y: Intercellular communication and the control of tissue growth: lack of communication between cancer cells. Nature 209: 1248-1249, 1966.

21. Naus CC and Laird DW: Implications and challenges of connexin connections to cancer. Nat Rev Cancer 10: 435-441, 2010.

22. Leithe E, Sirnes S, Omori Y and Rivedal E: Downregulation of gap junctions in cancer cells. Crit Rev Oncog 12: 225-256, 2006.

23. Hanna EA, Umhauer S, Roshong SL, et al: Gap junctional intercellular communication and connexin 43 expression in human ovarian surface epithelial cells and ovarian carcinomas in vivo and in vitro. Carcinogenesis 20: 1369-1373, 1999.

24. Rüttinger C, Bergmann M, Fink L, et al: Expression of connexin 43 in normal canine testes and canine testicular tumors. Histochem Cell Biol 130: 537-548, 2008.

25. Kanczuga-Koda L, Sulkowska M, Koda M, Rutkowski R and Sulkowski S: Increased expression of gap junction protein - connexin 32 in lymph node metastases of human ductal breast cancer. Folia Histochem Cytobiol 45 (Suppl 1): S175-S180, 2007.

26. Zhang W, DeMattia JA, Song H and Couldwell WT: Communication between malignant glioma cells and vascular endothelial cells through gap junctions. J Neurosurg 98: 846-853, 2003.

27. Saito-Katsuragi M, Asada H, Niizeki H, et al: Role for connexin 26 in metastasis of human malignant melanoma: communication between melanoma and endothelial cells via connexin 26. Cancer 110: 1162-1172, 2007.

28. Lavelle F, Bissery MC, Combeau C, Riou JF, Vrignaud P and André S: Preclinical evaluation of docetaxel (Taxotere). Semin Oncol 22: 3-16, 1995.

29. Harris AL: Emerging issues of connexin channels: biophysics fills the gap. Q Rev Biophys 34: 325-472, 2001.

30. Beyer EC, Paul DL and Goodenough DA: Connexin family of gap junction proteins. J Membr Biol 116: 187-194, 1990.

31. Srinivas M, Hopperstad MG and Spray DC: Quinine blocks specific gap junction channel subtypes. Proc Natl Acad Sci USA 98: 10942-10947, 2001.

32. Tao L and Harris AL: 2-aminoethoxydiphenyl borate directly inhibits channels composed of connexin26 and/or connexin 32. Mol Pharmacol 71: 570-579, 2007.

33. Maeda S, Nakagawa S, Suga M, et al: Structure of the connexin 26 gap junction channel at 3.5 A resolution. Nature 458: 597-602, 2009.

34. Bouvier D, Spagnol G, Chenavas S, et al: Characterization of the structure and intermolecular interactions between the connexin40 and connexin 43 carboxyl-terminal and cytoplasmic loop domains. J Biol Chem 284: 34257-34271, 2009. 